\title{
Study on Stress Solution Precision of the Plastic Zone near Mode-I Crack Line under Unidirectional Uniform Stress
}

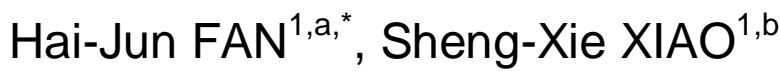 \\ ${ }^{1}$ School of Civil Engineering, Chongqing Jiaotong University, Chongqing, 400074

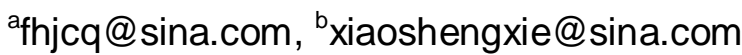 \\ ${ }^{*}$ Corresponding author
}

\begin{abstract}
Keywords: Mode-I Crack, Mohr-Coulomb Yield Criterion, Line Field Analytical Method, Stress General Solution of the Plastic Zone.
\end{abstract}

\begin{abstract}
For imprecise stress solution of the plastic zone near mode-I crack line of infinite wide plate under unidirectional uniform stress in relevant research literatures, this paper, by virtue of Mohr-Coulomb yield criterion and line field analytical method, re-infers and solves the stress general solution of the plastic zone satisfying equilibrium equation and yield criterion, as well as the stress solution and the elastic-plastic boundary of the plastic zone (scope of the plastic zone), points out unreasonable points in relevant research literatures, solves the similarities and differences about the stress solution of the plastic zone near mode-I crack line with Tresca yield criterion and Mohr-Coulomb yield criterion under unidirectional tension and press, and corrects imprecise results about the stress solution of the plastic zone near mode-I crack line of infinite wide plate under unidirectional uniform stress in relevant research literatures.
\end{abstract}

\section{Introduction}

Different from classical fracture theory which takes the stress field near the crack tip as the object of study, the analytical method of crack line field takes the stress field near the crack line as the object of study and solves the stress solution of the plastic zone through simultaneous equilibrium equation and selected yield criterion, which can effectively solve the problem that in a certain area near the crack line it is not restricted by small yield assumptions, and whose derivation process is of strict mechanical and physical significance, and in theory we can get infinitely precise solution of the whole crack elastic-plastic zone's stress values. As early as 1984, J. D. Aachenbach et al [1,2] first proposed the idea of line field analysis, in 1987, Guo Quanxin et al [3] developed this analytical method, and based on this, Yi Zhijian et al [4-6] proposed a complete line field analytical method, while Jian-hua Wang [7], Zhou Xiaoping [8-11], C. Guo [12], Y. X. Zhang [13], Guo JunHong [14], M. K. Huang [15], B. H. Zhang [16], Wang Cheng [17-18] et al developed research on theory and application with line field analytical method. Although the analytical method of crack line field, after thirty years of development, has made some valuable research achievements, there are still many problems not completely solved, limiting this method's development, application and promotion. For problems of mode-I crack of infinite wide plate under unidirectional uniform stress, there are main research literatures including: literatures [4] [6] research ideal elastic-plastic materials with Tresca yield criterion; literature [18] researches the damage of concrete micro-crack elastic and brittle static force under unidirectional tension with Mohr-Coulomb yield criterion, of which literatures [4] and [5] only get a group of particular solutions for the stress field of the plastic zone and think that simply by equilibrium equation and yield criterion we cannot get the stress general solution of the plastic zone, literature [6] proves that this conclusion is wrong through derivation, but the lack of one ordinary differential equation derived by yield criterion in literature [6] causes the problem that first order power term and higher order terms in the stress general solution of the plastic zone derived do not fully satisfy the yield criterion, and the general solution is not precise enough; literatures [17] and [18] follow the derivation process of literature [5] with line field analytical method, whose results are as unreasonable as that in literature [5]; literatures [7-11]cite the plastic zone stress general solution in literature [6] in the study on a pair or two pairs 
of concentrated force with Tresca yield criterion, resulting in imprecise derivation results. For imprecise stress solution of the plastic zone near mode-I crack line of infinite wide plate under unidirectional uniform stress in above relevant research literatures, this paper, by virtue of Mohr-Coulomb yield criterion, will re-infer and solve the stress solution and the elastic-plastic boundary (scope of the plastic zone) of the plastic zone near the crack line satisfying equilibrium equation and yield criterion, solve the similarities and differences about the stress solution of the plastic zone with Tresca yield criterion and Mohr-Coulomb yield criterion under tension and press, and fully correct imprecise results about the stress solution of the plastic zone near mode-I crack line of infinite wide plate under unidirectional uniform stress.

\section{Stress Solution of the Elastic Zone near the Crack Line}

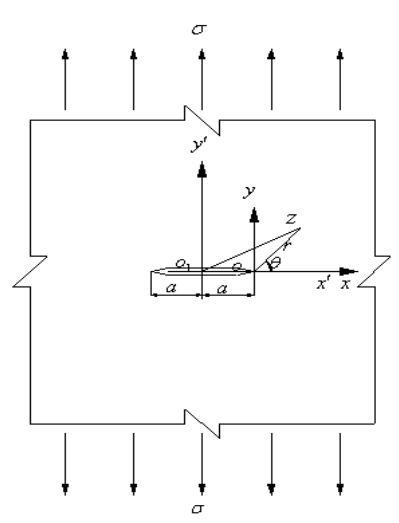

Fig. 1 Infinite Plate for Unidirectional Tension Stress Cracking on Mode-I Plane

For the elastic mechanical problem shown in Fig. 1, Using Westergaard stress function [4, 6, 7], the Stress expressions are:

$$
\begin{aligned}
& \sigma_{x}^{\mathrm{e}}=\operatorname{Re} Z-y \operatorname{Im} Z^{\prime}-\frac{\sigma}{2} \\
& \sigma_{y}^{\mathrm{e}}=\operatorname{Re} Z+y \operatorname{Im} Z^{\prime}+\frac{\sigma}{2} \\
& \tau_{x y}^{\mathrm{e}}=-y \operatorname{Re} Z^{\prime}
\end{aligned}
$$

Wherein:

$$
Z(z)=\frac{\sigma z}{\sqrt{z^{2}-a^{2}}}-\frac{\sigma}{2}
$$

Expand equation (1) at $\theta \rightarrow 0$ with Taylor series, then we can get the stress power series formal solutions of the elastic zone near the crack line as follows:

$$
\begin{aligned}
& \sigma_{x}^{\mathrm{e}}=\frac{\sigma}{\sqrt{r(2 a+r)}}\left[(a+r)-\frac{7 a^{3}+5 a^{2} r-3 a r^{2}}{2(2 a+r)^{2}} \theta^{2}\right]-\sigma+O\left(\theta^{4}\right) \\
& \sigma_{y}^{\mathrm{e}}=\frac{\sigma}{\sqrt{r(2 a+r)}}\left[(a+r)+\frac{5 a^{3}+7 a^{2} r+3 a r^{2}}{2(2 a+r)^{2}} \theta^{2}\right]+O\left(\theta^{4}\right) \\
& \tau_{x y}^{\mathrm{e}}=\frac{\sigma}{\sqrt{r(2 a+r)}}\left[\frac{a^{2}}{2 a+r} \theta-\frac{31 a^{4}-50 a^{3} r+28 a^{2} r^{2}}{6(2 a+r)^{3}} \theta^{3}\right]+O\left(\theta^{5}\right)
\end{aligned}
$$


Equation (1) can be infinitely expanded with Taylor series according to precision requirements, this paper herein takes $\theta$ 's third power term and ignores $\theta^{4}$ higher order term. Through Taylor expansion analysis in equation (3), first terms of $\sigma_{x}^{e}, \sigma_{y}^{y}$ are exact stress solutions on the classical stress intensity factor fracture theoretical crack line, namely a particular solution of equation (3) when $\theta=0$.

\section{Equilibrium Equation}

For the infinite wide plate with mode-I crack under unidirectional uniform tension shown in Fig. 1 , its equations of static equilibrium are:

$$
\begin{aligned}
& \frac{\partial \sigma_{x}}{\partial x}+\frac{\partial \tau_{x y}}{\partial y}=0 \\
& \frac{\partial \tau_{x y}}{\partial x}+\frac{\partial \sigma_{y}}{\partial y}=0
\end{aligned}
$$

\section{Mohr-Coulomb Yield Criterion}

According to Mohr-Coulomb theory, in consideration of principal stress intensity order $\sigma_{1} \geq \sigma_{2} \geq \sigma_{3}$, the yield criterion can be expressed as:

$$
\frac{1}{2}\left(\sigma_{1}-\sigma_{3}\right)+\frac{1}{2}\left(\sigma_{1}+\sigma_{3}\right) \sin \varphi-c \cos \varphi=0
$$

Wherein, $c$ and $\phi$ respectively refer to rock-soil cohesion and internal friction angle parameters.

From elastic mechanical stress positive and negative rules that "both positive and negative are positive", for the plane stress problem under unidirectional tension, the values of $\sigma 、 \sigma_{x}^{e} 、 \sigma_{y}^{e}$ in equation (3) are all greater than 0 , and in the vicinity of $\theta \rightarrow 0$, the first term of $\tau_{x y}^{e}$ relative to $\sigma_{x}^{e}$ and $\sigma_{y}^{e}$ is a high order minor term, namely $\tau_{x y}^{e} \prec \prec \sigma_{x}^{e} 、 \sigma_{y}^{e}$, so the principal stress intensity order is:

$$
\begin{aligned}
& \sigma_{1}=\frac{\sigma_{x}+\sigma_{y}}{2}+\sqrt{\left(\frac{\sigma_{x}-\sigma_{y}}{2}\right)^{2}+\tau_{x y}^{2}} \\
& \sigma_{2}=\frac{\sigma_{x}+\sigma_{y}}{2}-\sqrt{\left(\frac{\sigma_{x}-\sigma_{y}}{2}\right)^{2}+\tau_{x y}^{2}} \\
& \sigma_{3}=0
\end{aligned}
$$

Substitute equation (6) into Mohr-Coulomb yield criterion equation (5), we can get:

$$
\frac{\sigma_{x}+\sigma_{y}}{2}+\sqrt{\left(\frac{\sigma_{x}-\sigma_{y}}{2}\right)^{2}+\tau_{x y}^{2}}=\frac{2 c \cos \varphi}{1+\sin \varphi}
$$

Wherein, $c$ and $\varphi$ respectively refer to rock-soil cohesion and internal friction angle parameters.

To simplify the formula, please let $C_{t}=\frac{2 c \cos \phi}{1+\sin \phi}$.

For the plane stress problem under unidirectional uniform compression, the values of $\sigma 、 \sigma_{x}^{e}, \sigma_{y}^{e}$ in equation (3) are all less than 0, upon comparison of the principal stress intensity, we can get 
Mohr-Coulomb yield criterion as follows:

$$
\frac{\sigma_{x}+\sigma_{y}}{2}-\sqrt{\left(\frac{\sigma_{x}-\sigma_{y}}{2}\right)^{2}+\tau_{x y}^{2}}=-\frac{2 c \cos \varphi}{1-\sin \varphi}
$$

Wherein, $c$ and $\varphi$ respectively refer to rock-soil cohesion and internal friction angle parameters.

To simplify the formula, please let $C_{t}=\frac{2 c \cos \phi}{1+\sin \phi}$.

Compare equations (7) and (8) with Tresca yield criterion, as shown in the expression of Tresca yield criterion, i.e. $\tau_{\max }=\frac{\sigma_{1}-\sigma_{3}}{2}=k$ ( $k$ refers to the maximum shear stress limit), for the plane stress problem under unidirectional tension and press, in consideration of the principal stress intensity order, when we substitute it into Tresca yield criterion, the left side of the equation is consistent with the left sides of equations (7) and (8), but the constant term on the right side of the equation is of different physical significance, reflecting in that the yield stress of Mohr-Coulomb yield criterion is less than the yield stress of Tresca yield criterion.

\section{Stress General Solution of the Plastic Zone near the Crack Line}

In the plastic zone near the crack line, $\sigma_{x}$ and $\sigma_{y}$ are symmetric relative to the crack line, $\tau_{x y}$ is anti-symmetric relative to the crack line, the coordinate system is as shown in Fig. 1, so we can expand stress component at $y \rightarrow 0$ with Taylor series as follows:

$$
\begin{aligned}
& \sigma_{x}^{\mathrm{p}}=p_{0}(x)+p_{2}(x) y^{2}+O\left(y^{4}\right) \\
& \sigma_{y}^{\mathrm{p}}=q_{0}(x)+q_{2}(x) y^{2}+O\left(y^{4}\right) \\
& \tau_{x y}^{\mathrm{p}}=s_{1}(x) y+s_{3}(x) y^{3}+O\left(y^{5}\right)
\end{aligned}
$$

Substitute equation (9) into equilibrium equation (4), compare $y$ 's power, omit high order infinite minor terms above $y^{4}$, we can get:

$$
\begin{aligned}
& p_{0}^{\prime}(x)+s_{1}(x)=0 \\
& p_{2}^{\prime}(x)+3 s_{3}(x)=0 \\
& s_{1}^{\prime}(x)+2 q_{2}(x)=0
\end{aligned}
$$

Substitute equation (9) into yield criterion equation (7), compare y's power, omit high order infinite minor terms above $y^{4}$, upon simplification, we can get:

$$
\begin{aligned}
& \left(C_{t}-q_{0}\right)\left(C_{t}-p_{0}\right)=0 \\
& q_{2}\left(p_{0}-C_{t}\right)+p_{2}\left(q_{0}-C_{t}\right)=s_{1}^{2} \\
& p_{2} q_{2}=2 s_{1} s_{3}
\end{aligned}
$$

As shown in equations (10) and (11), when $q_{0}=C_{t}$ and $p_{0}$ is a constant less than $C_{t}$, we can get a group of particular solutions satisfying equilibrium equation and yield criterion: $q_{2}=p_{2}=s_{1}=s_{3}=\cdots=0$. According to the analysis in the previous section, the left side of Tresca yield criterion expression is the same as that of Mohr-Coulomb yield criterion expression, 
literatures [4] and [5] only get a group of particular solutions with Tresca yield criterion and an incorrect $r_{0}$ expression through elastic-plastic boundary matching, which think that simply by equilibrium equation and yield criterion we cannot get the stress general solution of the plastic zone, the derivation in literature [6] and this paper's follow-up derivation prove that this conclusion is wrong, but the lack of (12c) ordinary differential equation derived by yield criterion in literature [6] causes the problem that first order power term and higher order terms in the stress general solution of the plastic zone derived do not fully satisfy the yield criterion, hereby we need to point out that both literatures [17] and [18] follow the derivation process of literature [5] respectively in the study on the damage of concrete micro-crack elastic and brittle static force under unidirectional tension and the study on the crack under internal pressure of rock mass joint, whose results are as unreasonable as that in literature [5].

Simultaneous equations (10a), (10c) and (11b), substitute $q_{0}=C_{t}$ into the equation, we can get the ordinary differential equation general solution as follows:

$$
p_{0}=\frac{c_{1}}{x+c_{2}}+C_{t}
$$

Substitute equation (12) into equations (10a) and (10c), we can get:

$$
\begin{aligned}
& s_{1}=\frac{c_{1}}{\left(x+c_{2}\right)^{2}} \\
& q_{2}=\frac{c_{1}}{\left(x+c_{2}\right)^{3}}
\end{aligned}
$$

Simultaneous equations (10b) and (10c), substitute equations (13) and (14) into the equation, we can get:

$$
\begin{gathered}
p_{2}=c_{3}\left(\left|x+c_{2}\right|\right)^{-\frac{3}{2}} \\
s_{3}=\frac{c_{3}}{2}\left(\left|x+c_{2}\right|\right)^{-\frac{5}{2}}
\end{gathered}
$$

Substitute $q_{0}=C_{t}$ and equations (12)-(16) into equation (9), we can get the stress general solutions of the plastic zone near the crack line as follows:

$$
\begin{aligned}
& \sigma_{x}^{\mathrm{p}}=\frac{c_{1}}{x+c_{2}}+C_{t}+\frac{c_{3}}{\left(\left|x+c_{2}\right|\right)^{\frac{3}{2}}} y^{2}+O\left(y^{4}\right) \\
& \sigma_{y}^{\mathrm{p}}=C_{t}+\frac{c_{1}}{\left(x+c_{2}\right)^{3}} y^{2}+O\left(y^{4}\right) \\
& \tau_{x y}^{\mathrm{p}}=\frac{c_{1}}{\left(x+c_{2}\right)^{2}} y+\frac{c_{3}}{2\left(\left|x+c_{2}\right|\right)^{\frac{5}{2}}} y^{3}+O\left(y^{5}\right)
\end{aligned}
$$

Wherein, $c_{1}, c_{2}$ and $c_{3}$ are undetermined coefficients.

Equation (17) is the general solution expression of $y$ 's third order power term and lower order power terms strictly satisfying equilibrium equation and yield criterion, namely satisfying equations (10) and (11) at the same time. As shown in the above general solution derivation process, if you want to solve $y^{4}$ and higher order power terms, you only need to expand the plastic zone stress equation (9) into high order multiple-term equation with Taylor series and substitute it into equilibrium equation and yield criterion, compare $y$ 's power, equations (10) and (11) will increase 
corresponding equations, increased number of equations is the same as the number of unknown terms, and the general solution can be obtained by solving the system of ordinary differential equations, namely that the stress power series form general solution of the plastic zone near the crack line can be solved to $y$ 's any power high order.

For solving the plane stress under unidirectional compression with Mohr-Coulomb yield criterion, we only need to select yield criterion equation (8), simultaneous equilibrium equation, substitute it into the above general solution solving process, and change $C_{t}$ into $C_{c}$, we can get the general solution expression the same as equation (17), specific derivation process will not be repeated here.

In the study on the plane stress problem under unidirectional tension and press, the left side of Tresca yield criterion expression is consistent with the left side of equation (7), we only need to change $C_{t}$ into $2 k$, and the expression for the stress general solution of the plastic zone obtained is the same, specific derivation process will not be repeated here. In this paper, the zero-order power term of general solution expression $(17 \mathrm{a}, \mathrm{b})$ and the first-order power term of equation $(17 \mathrm{c})$ are the same as that in literature [6]. However, we must point out that the zero-order power term and the first-order power term obtained in literature [6] can only satisfy equilibrium equation and partial Tresca yield criterion, while second-order power term and higher order terms $p_{2} 、 q_{2} 、 s_{1} 、 s_{3}$ do not satisfy equation (11c), namely not fully satisfying yield criterion, and the general solution is imprecise. Therefore, literatures [7-11] cite the plastic zone stress general solution shown in literature [6], whose results of derivation are imprecise.

To match with the elastic zone stress component equation (3), we need to change $x, y$ expressed in equation (17) into $\theta, r$ through coordinate transformation. Substitute $x=r \cos \theta$ and $y=r \sin \theta$ into equation (17), expand it at $\theta \rightarrow 0$ with Taylor series, omit high order infinite minor terms above $y^{4}$, we can get:

$$
\begin{aligned}
& \sigma_{x}^{\mathrm{p}}(r, \theta)=\frac{c_{1}}{r+c_{2}}+C_{t}+\left\lfloor\frac{c_{1} r}{\left(r+c_{2}\right)^{2}}+\frac{2 c_{3} r^{2}}{\left(\left|r+c_{2}\right|\right)^{\frac{3}{2}}}\right\rfloor \theta^{2}+O\left(\theta^{4}\right) \\
& \sigma_{y}^{\mathrm{p}}(r, \theta)=C_{t}+\frac{2 c_{1} r^{2}}{\left(r+c_{2}\right)^{3}} \theta^{2}+O\left(\theta^{4}\right) \\
& \tau_{x y}^{\mathrm{p}}(r, \theta)=\frac{c_{1} r}{\left(r+c_{2}\right)^{2}} \theta+\left\lfloor\frac{6 c_{1} r^{2}}{\left(r+c_{2}\right)^{3}}-\frac{c_{1} r}{\left(r+c_{2}\right)^{2}}+\frac{3 c_{3} r^{3}}{\left(\left|r+c_{2}\right|^{\frac{5}{2}}\right.}\right\rfloor \theta^{3}+O\left(\theta^{4}\right)
\end{aligned}
$$

Wherein, $c_{1}, c_{2}$ and $c_{3}$ are undetermined coefficients.

\section{Elastic-Plastic Boundary near the Crack Line and Matching Results}

Elastic-plastic boundary $r=r_{\mathrm{p}}(\theta)$ is symmetric relative to the crack line, expand elastic-plastic boundary $r=r_{\mathrm{p}}(\theta)$ at $\theta \rightarrow 0$ with power series as follows [3]:

$$
r_{\mathrm{p}}=r_{0}+r_{2} \theta^{2}+O\left(\theta^{4}\right)
$$

Wherein: $r_{0}$ refers to the length of the plastic zone on the crack line, while $r_{0}$ and $r_{2}$ are undetermined coefficients.

Substitute equation (19) into equation (18), we can get the Taylor series expansion equation of the plastic zone stress on the elastic-plastic boundary at $\theta=0$ as follows: 


$$
\begin{aligned}
& \sigma_{x}^{\mathrm{p}}\left(r_{0}, \theta\right)=\frac{c_{1}}{r_{0}+c_{2}}+C_{t}+\left\lfloor\frac{c_{1} r_{0}}{\left(r_{0}+c_{2}\right)^{2}}+\frac{2 c_{3} r_{0}^{2}}{\left(\mid r_{0}+c_{2}\right)^{\frac{3}{2}}}-\frac{c_{1} r_{2}}{\left(r_{0}+c_{2}\right)^{2}}\right\rfloor \theta^{2}+O\left(\theta^{4}\right) \\
& \sigma_{y}^{\mathrm{p}}\left(r_{0}, \theta\right)=C_{t}+\frac{2 c_{1} r_{0}{ }^{2}}{\left(r_{0}+c_{2}\right)^{3}} \theta^{2}+O\left(\theta^{4}\right) \\
& \tau_{x y}^{\mathrm{p}}\left(r_{0}, \theta\right)=\frac{c_{1} r_{0}}{\left(r_{0}+c_{2}\right)^{2}} \theta+\left\lfloor\frac{6 c_{1} r_{0}{ }^{2}-2 c_{1} r_{0} r_{2}}{\left(r_{0}+c_{2}\right)^{3}}+\frac{c_{1}\left(r_{2}-r_{0}\right)}{\left(r_{0}+c_{2}\right)^{2}}+\frac{3 c_{3} r_{0}{ }^{3}}{\left(\left|r_{0}+c_{2}\right|\right)^{\frac{5}{2}}}\right\rfloor \theta^{3}+O\left(\theta^{4}\right)
\end{aligned}
$$

In a similar way, substitute equation (19) into equation (3), we can get the elastic zone stress as follows:

$$
\begin{aligned}
& \sigma_{x}^{\mathrm{e}}=\frac{\sigma}{\sqrt{r_{0}\left(2 a+r_{0}\right)}}\left\{\left(a+r_{0}\right)-\left[\frac{7 a^{3}+5 a^{2} r_{0}-3 a r_{0}^{2}}{2\left(2 a+r_{0}\right)^{2}}+\frac{r_{2} a^{2}}{r_{0}\left(2 a+r_{0}\right)}\right] \theta^{2}\right\}-\sigma+O\left(\theta^{4}\right) \\
& \sigma_{y}^{\mathrm{e}}=\frac{\sigma}{\sqrt{r_{0}\left(2 a+r_{0}\right)}}\left\{\left(a+r_{0}\right)+\left[\frac{5 a^{3}+7 a^{2} r_{0}+3 a r_{0}^{2}}{2\left(2 a+r_{0}\right)^{2}}-\frac{r_{2} a^{2}}{r_{0}\left(2 a+r_{0}\right)}\right] \theta^{2}\right\}+O\left(\theta^{4}\right) \\
& \tau_{x y}^{\mathrm{e}}=\frac{\sigma}{\sqrt{r_{0}\left(2 a+r_{0}\right)}}\left\{\frac{a^{2}}{2 a+r_{0}} \theta-\left[\frac{r_{2} a^{2}\left(a+2 r_{0}\right)}{r_{0}\left(2 a+r_{0}\right)}+\frac{31 a^{4}-50 a^{3} r_{0}+28 a^{2} r_{0}^{2}}{6\left(2 a+r_{0}\right)^{3}}\right] \theta^{3}\right\}+O\left(\theta^{5}\right)
\end{aligned}
$$

The matching conditions for the elastic zone and the plastic zone on the elastic-plastic boundary near the crack line are: they should meet continuity conditions on the elastic-plastic boundary, namely the normal component at any a point along the boundary of the elastic stress field and the plastic stress field should be the same, so does the tangential component. Solve the unit normal component at any a point on the elastic-plastic boundary near the crack line with equation (19), substitute into normal and tangential stress formula [5], compare $\theta$ 's power, upon simplification and decoupling, we can get [5]:

$$
\begin{gathered}
p_{n}^{e}=p_{n}^{\mathrm{p}} \\
q_{n}^{e}=q_{n}^{\mathrm{p}} \\
s_{n}^{e}=s_{n}^{\mathrm{p}}
\end{gathered}
$$

Wherein: $n=0,1,2,3, \cdots$

Substitute equations (20) and (21) into equation (22), we can solve undetermined coefficients as follows:

$$
\begin{aligned}
& r_{0}=a\left(\sqrt{\frac{C_{t}^{2}}{C_{t}^{2}-\sigma^{2}}}-1\right) \\
& r_{2}=\frac{2 r_{0}^{2} a^{2}}{\left[r_{0}\left(2 a+r_{0}\right)\right]^{\frac{3}{2}}}+\frac{r_{0}\left(5 a^{2}+7 a r_{0}+3 r_{0}^{2}\right)}{2 a\left(2 a+r_{0}\right)} \\
& c_{1}=\frac{\sigma r_{0}\left(2 a+r_{0}\right) \sqrt{r_{0}\left(2 a+r_{0}\right)}}{a^{2}}
\end{aligned}
$$




$$
\begin{aligned}
& c_{2}=\frac{-r_{0}\left(2 a+r_{0}\right) \sqrt{r_{0}\left(2 a+r_{0}\right)}}{a^{2}}-r_{0} \\
& c_{3}=-\frac{\sigma}{2}\left[\frac{a^{2}\left[r_{0}\left(2 a+r_{0}\right)\right]^{\frac{3}{4}}}{r_{0}}+\frac{7 a^{3}+5 a^{2} r_{0}-3 a r_{0}^{2}}{2\left[r_{0}\left(2 a+r_{0}\right)\right]^{\frac{1}{4}}}\right]
\end{aligned}
$$

Substitute equations (23a) and (23b) into equation (19), namely the elastic-plastic boundary. Substitute equations (23a) and (23c)-(23e) into equation (17), namely the stress solution of the plastic zone near the crack line, the expressions of equations $(23 a, c, d)$ are consistent with those in literature [6]. The first-order power term and higher order terms of the stress general solution of the plastic zone in literature [6] do not strictly satisfy yield criterion, the elastic-plastic boundary matching equation is different, so the expressions of equations $(23 \mathrm{~b}, \mathrm{e})$ are inconsistent with those in literature [6], but this paper adopts the plastic zone stress general solution strictly satisfying yield criterion to solve undetermined coefficients, so the physical and mechanical relationship is more precise.

Obviously, through elastic-plastic boundary decoupling matching, for the plane stress problem under unidirectional uniform tension and press, the expression for undetermined coefficients solved with Mohr-Coulomb yield criterion and Tresca yield criterion only needs to change $C_{t}$ in equation (23a) respectively into $C_{c}$ and $2 k$, the expressions for other undetermined coefficients are the same, specific derivation process will not be repeated here

\section{Conclusion}

(1)This paper points out imprecise stress solution of the plastic zone near the crack line in relevant research literatures, re-infers the plastic stress solution near mode-I crack of infinite wide plate under unidirectional uniform tension with Mohr-Coulomb yield criterion, solves the stress general solution of the plastic zone satisfying equilibrium equation and yield criterion, as well as the stress solution and the elastic-plastic boundary of the plastic zone (scope of the plastic zone), and corrects imprecise results about the stress solution of the plastic zone in relevant research literatures.

(2)Under unidirectional uniform tension and press, the expressions of Mohr-Coulomb yield criterion are somewhat different, but the expressions for the stress solution and the stress general solution of the plastic zone near mode-I crack line are the same.

(3)Under unidirectional uniform tension and press, the expressions for the stress solution and the stress general solution of the plastic zone near mode-I crack line with Mohr-Coulomb yield criterion and Tresca yield criterion simultaneous equilibrium equation are the same, with difference only in material's physical constant.

(4)For various materials satisfying Mohr-Coulomb yield criterion and Tresca yield criterion, with line field analytical method, the stress solution of the plastic zone near mode-I crack line can be exactly solved to $y$ 's any power high order, and all order power terms will strictly satisfy equilibrium equation and yield criterion.

\section{References}

[1]J.D.Achenbach, V.Dunayevsky, Crack growth under plane stress conditions in an elastic-perfectly-plastic materia, J. Mechanics and Physics of Solids. 32(1984)89-100.

[2]J.D. Achenbach, Z.L. Li, Plane crack-line fieds for crack growth in an elastic- perfectly-plastic material, Engineering Fracture Mechanics. 20(1984)535-544.

[3]Guo Quanxin, Li Kerong, Plastic deformation ahead of a plane stress tensile crack growing in an elastic-perfectly-plastic solid, Engineering Fracture Mechanics. 28(1987)139-146. 
[4]Zhi-jian Yi, The near crack line solutions for plane stress tensile crack growth in an elastic-perfectly plastic matierial, Engineering Fracture Mechanics. 42(1992)169-176.

[5]Yi Zhijian, Wang Shijie, Exact solutions of near crack line fields for mode I crack under plane stress condition in an elastic-perfectly plastic solid, Applied Mathematics and Mechanics. 17(1996)335-342.

[6]Yi zhijian, Yanbo, General form of matching equation of elastic-plastic field near crack line for mode I crack under plane stress condition, Applied Mathematics and Mechanics. 22(2001) 1173-1182.

[7]Jian-hua Wang, Xiao-ping Zhou, Near crack line elastic-plastic analysis for a infinite plate loaded by two pairs of point tensile forces, Mechanics Research Communications. 21(2004)415-420.

[8]Zhou Xiaoping, Zhang Yongxing, Zhu Keshan, Elastic-plastic analytical solution for an eccentric crack loaded plate by a pair of tensile point forces, Acta Mechanica Solida Sinica.26(2005)267-272.

[9]Xiao-ping ZHOU, Tong-hua LING, Elastic-plastic analytical solution for centric crack loaded by two pairs of point shear forces in finite plate, Transactions of Nonferrous Metals Society of China. 16(2006)1009-1014.

[10]Zhou Xiaoping, Yang Haiqing, Zhang yongxing, Elastoplastic analysis of a finate plate with an eccentric crack loaded by two pair of concentrated tensile forces, Engineering Mechanics. 25(2008)22-27.

[11]X.P. Zhou, H.Q. Yang, Elastoplastic solution for an eccentric crack loaded by two pairs of point tensile forces, Theoretical and Applied Fracture Mechanics., 51(2009) 62-72.

[12]C. Guo, X.P. Zhou, Y.X. Zhang, H.Q. Yang, X.H. Li, Elastic-plastic near field solution of an eccentric crack under shear in a finite width plate,Theoretical and Applied Fracture Mechanics. 51(2009)174-180.

[13]Y.X. Zhang, X.P. Zhou, H.Q. Yang, X.H. Li, M.K. Huang, Q. Xie, Elastoplasticity of crack loaded by two pairs of isolated shear forces in finite width plate, Theoretical and Applied Fracture Mechanics.54(2010)141-148.

[14]Guo Junhong, Lu Zixing, Line field analysis and complex variable method for solving elastic-plastic fields around an anti-plane elliptic hole, Science China Physics, Mechanics\& Astronomy. 54(2011)1495-1501.

[15]M.K. Huang, Y.B. Huang, R.C. Tian, Q. Xie,Elastoplastic near field solution for mode II crack loaded by remote shear stress in an infinite plate, Theoretical and Applied Fracture Mechanics. 55(2011)222-226.

[16]B.H. Zhang, J.H. Deng, W.D. Wu, J.J. Liu, Mode I crack in an elasto-perfectly plastic material under pore water pressure of a finite medium, Theoretical and Applied Fracture Mechanics. 57(2012)31-35.

[17]Wang cheng, Deng anfu, Elastic-brittle fracture analysis of micro-crack in concrete loaded by stretching stresses, Journal of Chongqing Jianzhu University. 21(1999)66-69.

[18]Wang cheng, Deng anfu, Analytical study on fracture of jointed rock mass under internal pressures, Chinese Journal of Rock Mechanics and Engineering. 21(2002)640-643. 